\title{
Torsional force applied to the tibia of living lambs in an attempt to change the bone rotational axis ${ }^{1}$
}

\author{
José Batista Volpon', Maurício José Falcai ${ }^{\mathrm{II}}$, Carlos Alberto Moro ${ }^{\mathrm{III}}$, Daniel Mendes Leal ${ }^{\mathrm{IV}}$ \\ ${ }^{I} \mathrm{PhD}$, Full Professor, Department of Biomechanics, Medicine and Rehabilitation of the Locomotor System, School of Medicine of Ribeirao Preto, \\ University of Sao Paulo (USP), Brazil. Design, intellectual and scientific content of the study, interpretation of data, manuscript writing, critical \\ revision.

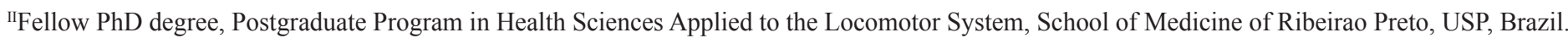 \\ Animal care, technical procedures. ${ }^{\text {III} M a s t e r, ~ E n g i n e e r, ~ L a b o r a t o r y ~ o f ~ B i o e n g i n e e r i n g, ~ S c h o o l ~ o f ~ M e d i c i n e ~ o f ~ R i b e i r a o ~ P r e t o, ~ U S P, ~ B r a z i l . ~ T o r s i o n ~}$ \\ device design and mechanical testing performance.

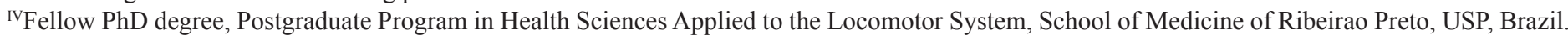 \\ Animal care, technical procedures, critical revision.
}

\section{ABSTRACT}

PURPOSE: To evaluate the effects of torsional force on the rotational axis of living lamb tibias.

METHODS: An external fixator device was designed to apply rotation to the tibias of lambs. Once a week, the bone distal extremity was rotated $2^{\circ}$. After achieving $\sim 20^{\circ}$ of internal rotation, the turning was discontinued and the device was maintained in situ for one month and euthanasia occurred in group $A(n=10)$ after this. In group $B(n=9)$ euthanasia occurred three months after removing the device. Computed tomography scans evaluated the rotational angle; dual-energy X-ray absorptiometry assessed the bone mineral density, and conventional and polarized light microscopy studied the bone microstructure.

RESULTS: In group A, the mean angle of the external rotation in the control tibias was $24^{\circ}$ and $8^{\circ}$ in the twisted tibias ( $<<0.0001$ ); in group $B$, the angle was $23^{\circ}$ (control) and $7^{\circ}$ (twisted, $p<0.0001$ ), with no differences between groups $A$ and $B$ ( $p=0.9567$ ). The BMD increased in the twisted tibias in group A $(p<0.0001)$ and in group $B(p=0.0023)$, with no between-group differences $(p>0.05)$. Microscopically, the twisted tibias showed asymmetrical subperiosteal bone deposition on the lateral cortex surface.

CONCLUSION: Gradual torsion applied to the immature tibia significantly modified its rotational axis.

Key words: Orthopedic Fixation Devices. Tibia. Bone Malalignment. Torsion, Mechanical. Sheep. 


\section{Introduction}

Growth affects not only body size and weight but also the somatic configuration through changes in posture and in the axial alignment of the different anatomical segments of the musculoskeletal system.

A child's rotational profile of the lower limbs is a good illustration of the dynamic modifications that take place as growth evolves. At birth, the femur and tibia are anatomically excessively rotated inwardly. This association confers to the young normal child a pattern of gait with the lower limbs facing medially. This excessive rotation gradually decreases as the child grows ${ }^{1}$ resulting in the normal gait pattern in which both feet are slightly pointing outward. However, in a few otherwise normal children, the immature gait pattern may persist, and a lower limb rotational malalignment results. The rotational profile may also be affected by underlying pathologic conditions that apply abnormal forces to the immature bones. The excessive femoral internal rotation and external tibial rotation in children with cerebral palsy are good examples of the interaction between the adverse mechanical demands and the bone morphology ${ }^{2}$.

The physiologic developmental rotational profile is still not completely understood, but it certainly results from the combined effect of intrinsic and environmental factors. Moreland applied torsional forces using plaster casts in the tibias of growing rabbits and was able to cause abnormal rotational changes. However, these changes took place at the growth plate and not along the body of the bone. He found no response at the level of the cortical bone but noted that the application of direct torsional loading to the bone shaft itself for longer periods of time might elicit some alteration in the rotational axis. Barlow and Staheli ${ }^{4}$ splinted the hind limbs of young rabbits in external rotation and three weeks later detected changes in the limb axial alignment; however, such changes occurred in the joint relationship and not at the bone's structural level.

In patients, when the rotational deformity demands correction, this correction is usually carried out by osteotomies that may be performed at different bone levels with stabilization by internal or external fixation methods ${ }^{5-7}$. However, osteotomies of the tibia have a high incidence of complications, such as nerve injury, difficult fixation, vascular complication and asymmetrical corrections $^{6-8}$. Consequently, the search for less invasive and more effective rotational misalignment corrections is justified.

We report the development and testing of a device that is specially designed to apply progressive torsional forces directly to the tibia of immature sheep without performing osteotomy. The main objective was to study the effect of such cumulative forces on the bone's rotational axis.

Our hypothesis is that applying cumulative torsional forces to the living immature tibia may cause a progressive and safe deformation that could lead to a significant modification of the bone's rotational axis. This modification may be permanently incorporated in to the bone's anatomy, with the future possibility of clinical use of a safer and well-controlled method to correct rotational malalignment.

\section{Methods}

The experimental protocol for this investigation was approved by the local Committee on Research and Animal Experimentation ( ${ }^{\circ}$ 004/2011).

The investigation was performed in three stages. First, the mechanical torsional profile of immature sheep tibias was obtained. Second, a mechanical torsional device was developed. Third, this device was applied to the tibia of the immature sheep.

\section{Tibia torsional mechanical profile}

Fifteen tibias were obtained from 6-month-old healthy sheep and cleaned of soft tissues. Then, each bone extremity was transfixed with two crossed $2.0 \mathrm{~mm}$ Kirschner wires and potted with methyl methacrylate cement to form a cylindrical block. This procedure is necessary to fix the specimen to the mechanical testing machine. These specimens were mechanically tested in a torsion machine (Instron 55 MT, Norwood, MA, USA) that was equipped with a $220 \mathrm{Nm}$ load cell. The proximal segment of the tibia was kept fixed, and internal rotation was applied to the distal extremity at an angular speed of $10 \% \mathrm{~min}$ until the bone fractured. A real-time torque versus angular deformation curve was obtained, and the ultimate torque and ultimate angular deformation were determined.

\section{Rotational device}

A torsional device was designed and manufactured using stainless steel with two attached components to ensure that one extremity could be turned in relation to the other. The major characteristics of this device are presented in Figure 1. Briefly, there were two aligned and interconnected cylindrical axes that could rotate independently. The connection between these cylinders was made using a mechanical joint that was linked to an intermediary telescopic shaft formed by gliding and rotating pieces. Rotation was provided by a central piece that contained a 
worm gear connected to the main teleshaft. Turning was applied manually with a removable key fitted into a pin of the worm gear. The extremities of the central shaft were built in such a manner that the rotation of only one segment was permitted as the gear was turned. At the ends of the shafts, two stainless steel blocks were attached. These blocks contained two holes for the future insertion of metallic pins to be screwed into the lamb tibia, which firmly attached the device to the bone (Figure 1).

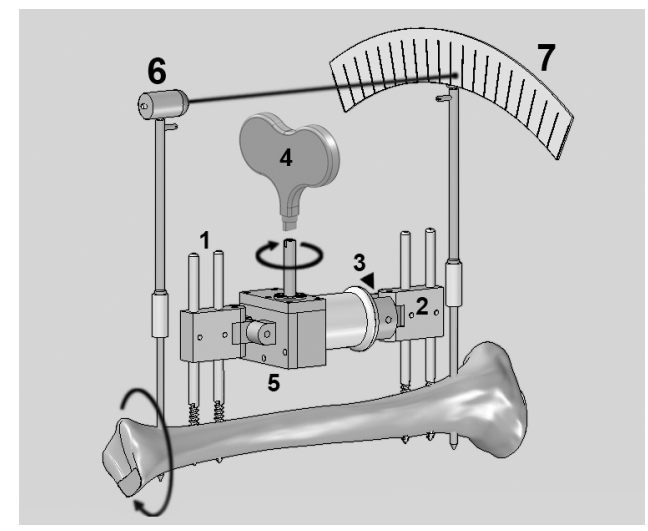

FIGURE 1 - Schematic of the rotational device applied to the tibia. The apparatus is fixed to the bone with Schanz screws (1) that are held by metal blocks (2) connected to rotating cylinders (3). Turning is performed using a "T" piece that is fitted to a pin (4) to ensure that rotation is transmitted to a worm gear (5), which rotates the extremities of a central shaft in opposite directions. Another removable device is temporarily fitted at the bone extremities to control the amount of rotation of the bone's distal extremity. This device has a laser beam source (6) attached to the distal extremity of the tibia and is projected against a scale on the proximal extremity (7).

\section{Rotation recorder}

When torsion was applied, the amount of rotation that occurred between the bone extremities was measured by a specially designed device that was formed from two independent pieces. The distal piece had a $2.8 \mathrm{~mm}$ metallic pin with a laser beam source in one extremity. The opposite extremity of the same pin had a hexagonal shape with dimensions to be firmly fitted into the hexagonal hole of the head of a $3.5 \mathrm{~mm}$ orthopedic screw inserted in the distal extremity of the tibia.

The proximal piece of the rotation recorder had a similar metallic pin with one extremity having a hexagonal tip. The opposite extremity of this pin was connected to a metallic plate that had marked divisions in degrees. This measuring recorder was used to control the amount of rotation of the distal tibia during the application of torsion and was removed after each turning session. The orthopedic screws used to fit the rotation recorder remained inserted in the tibial extremities with their head extruding through the skin.

\section{In vivo experiment}

Twenty crossbred 6-month-old male healthy lambs with a mean weight of $24 \mathrm{~kg}$ (standard deviation $[\mathrm{SD}]=1.50$ ) were used. Following a 24-hour fasting period, each animal received intravenous acepromazine $1 \%(0.1 \mathrm{mg} / \mathrm{kg})$, ketamine chloride $10 \%(2.0 \mathrm{mg} / \mathrm{kg})$ and xylazine $2 \%(0.05 \mathrm{mg} / \mathrm{kg})$. The anesthesia was completed with right sciatic nerve block (16.0 mg/kg Xylocaine). Then, the right hind limb was routinely prepared for surgery and draped freely.

The torsion apparatus was first connected to a metallic guide to facilitate its manipulation, positioning and correct alignment with the long axis of the left tibia. Then, one selfthreading $4.0 \mathrm{~mm}$ Schanz screw was inserted through the most proximal hole of the torsion device and introduced into the medial side of the metaphysis. Another Schanz screw was similarly introduced into the distal metaphysis. The correct alignment of the device along the tibial longitudinal axis was verified; another Schanz screw was introduced proximally, and another screw was placed distally to complete the device fixation (four pins). Still using the metallic guide, two $3.5 \mathrm{~mm}$ cortical orthopedic screws were inserted at the extremities: one proximal and one distal from the torsion apparatus. The screw heads were left extruding the skin with the purpose for future fitting to the rotation recorder.

The guide was removed, a radiograph was obtained, and the device was protected with bandages. An intravenous (IV) dose of hydrochloride $4.0 \mathrm{mg} / \mathrm{kg}$ tramadol was injected. The animal was allowed to recover from anesthesia in a separate box and then transferred to a collective cage (four animals each). Intramuscular injections of ketoprofen $(2.0 \mathrm{mg} / \mathrm{kg})$ were administered every 12 hours for the first two days.

\section{Post-operative follow-up}

The first session of rotation started 1 week post-surgery and was scheduled to occur once a week thereafter. Under gentle manual restraint and without using anesthetics, the lamb was manually held, and the torsional device was exposed. Following this process, the two pieces of the removable angular measuring device were assembled by inserting the hexagonal tips of their pins into the hexagonal holes of the screw heads that had been left extruding the skin. The laser beam from the distal piece was turned on and adjusted to the " 0 " position on the opposite angular scale. Using the appropriate removable key, the distal extremity of the torsion device was carefully rotated internally $2^{\circ}$. Afterward, both parts of the measuring device were removed, the dressings were replaced, and the animal was returned to the cage. The interruption 
of the torsion sessions occurred when the distal end of the tibia reached a final $20^{\circ}$ of internal rotation in relation to the initial angle. Then, the rotation device was kept in place for an additional month in all animals, but no twisting was applied during this period of time. After this process, two groups were created with the animals randomly distributed. In group $A(n=10)$, euthanasia occurred after one month, and the torsion device was removed. In group $B(n=9)$, after one month, the animals were anesthetized, the torsion device was removed, and the lamb was set free in a small pasture for three additional months. After completing that period of time without the device, the lamb was euthanized. In both groups, euthanasia was performed with an IV overdose of sodium pentobarbital followed by neck laceration, which allowed for exsanguination'. Following the animal's death, both tibias were collected and cleaned of soft tissues.

\section{Evaluation}

To measure the degree of rotation, each pair of tibias of the same animal were subject to a computed tomography scan (CT, the Brilliance CT 16-slice system, Phillips, Andover, MA, USA) with $8.0 \mathrm{~mm}$ thick transverse sections along the entire tibia. The transverse axis of a representative cut from the proximal and another cut from the distal tibial epiphyses were chosen, and the angle between them was measured. This measurement represents the angle of rotation of the distal extremity in relation to the proximal extremity. The procedure was performed by a radiologist who specialized in the locomotor system and who was not involved with the investigation.

Bone mineral density (BMD) was determined along a 1.0 $\mathrm{cm}$ length of bone at the mid-diaphysis of each pair of tibias using the dual-energy absorptiometry technique (DXA, Lunar - X-ray Bone Densitometer, DPX-alpha with SmartScan, version 4.7, Madison, WI, USA).

For the histological analysis, a $2.0 \mathrm{~cm}$ long cylinder of bone was excised from the mid-diaphysis. This bone block was defatted in $4 \%$ formaldehyde (48 hours) followed by dehydration in successive passages in increasing concentration of ethyl alcohol solutions. The specimens were embedded in acrylic resin (LR White resin, Sigma Aldrich, St. Louis, MO, USA) for 14 days, during which time the resin was changed daily. Ten $200 \mu \mathrm{m}$ thick slices were cut using a metallographic cutter (Büehler, IL, USA), and each specimen was reduced to a thickness of $10 \mu \mathrm{m}$ through grinding and polishing (Exart Grinding Systems, Oklahoma City, OK, USA). The specimens were stained in toluidine blue and examined under microcopy using ordinary and polarized light (Microscope Axio Imager M2, Carl Zeiss, Germany). Partial field photographs were acquired using a 2.5x objective (Axio Cam MRm Rev.3 FireWire) to ensure that the entire area of the specimen was documented. From several images, a single digital image of the whole section was created using the AxioVision Rel. 4.8.2 software (Carl Zeiss International).

\section{Statistical analysis}

For the statistical analyses, the software programs GraphPad and Prism version 5.00 (San Diego, CA, USA) were used. A paired Student's t-test for parametric data was applied to compare the twisted tibia to the control. Student's t-test was used to compare the results between groups $\mathrm{A}$ and $\mathrm{B}$, and $\mathrm{p}<0.05$ was considered to be statistically significant.

\section{Results}

\section{Mechanical torsion of the isolated tibias}

The mechanical tests of the isolated tibias obtained from normal animals showed a torsion profile with a mean maximum angular deflection of $17.26^{\circ}(\mathrm{SD}=1.41)$ and a mean maximum torque of $40.21 \mathrm{Nm}(\mathrm{SD}=4.2)$. In all cases, when the ultimate load was reached, spiral fractures occurred in the distal portion of the diaphysis with a segmental fragment in four cases.

In the in vivo experiment stage, the lambs subject to the operation recovered from the surgical procedure without complications. The animals started walking the day following the operation and soon resumed feeding and normal cage activities. Apparently, the twisting process was not painful, as the lambs did not present with limping, were appreciably mobile and bore weight on the operated limb. There was no swelling in the operated limb and no cases of infection. However, in group B, one animal's control tibia broke the first week after the device was removed; this animal was excluded from the study.

\section{Rotational angle of the tibias}

The mean time to reach $\sim 20^{\circ}$ of external rotation decrease was ten weeks.

The difference in rotation of the distal segment of the twisted tibia was perceived macroscopically at the end of the torsion process (Figure 2).

In group $\mathrm{A}$, the $\mathrm{CT}$ measurement of the rotation angle showed that the control tibias had an average external distal rotation of $24^{\circ}$ (range, $20^{\circ}$ to $26^{\circ} ; \mathrm{SD}=2$ ). The twisted tibias 
presented a mean external rotation of $8^{\circ}$ (range, $1^{\circ}$ to $10^{\circ} ; \mathrm{SD}=1$; $\mathrm{p}<0.0001$ ), which is a $66.7 \%$ decrease.

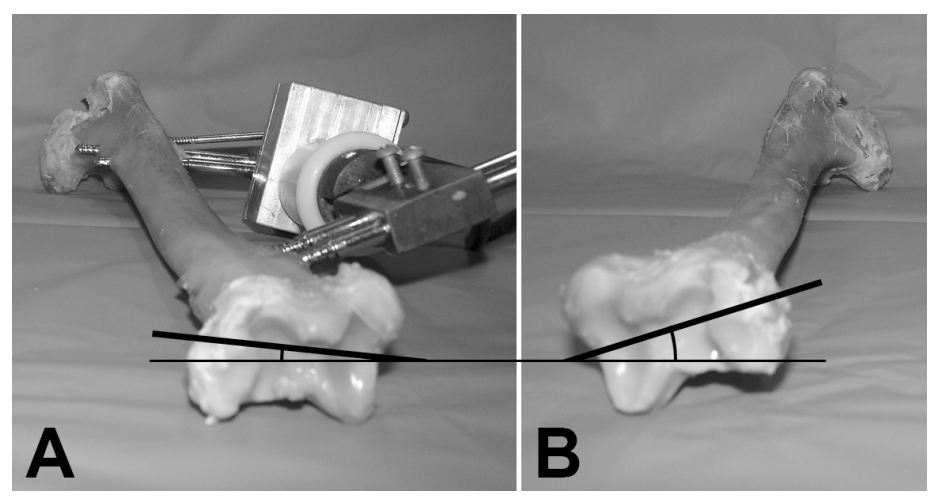

FIGURE 2 - Representative example of the twisted (A) and the control tibias (B). Note that the torsion device is in situ as well as the visual difference of the rotation angle of the distal extremity between the treated and the control bones.

In group $\mathrm{B}$, the distal rotation angle of the control tibias had an average external torsion of $23^{\circ}$ (range, $18^{\circ}$ to $27^{\circ}$; $\mathrm{SD}=3$ ). The twisted tibias presented a mean external rotation of $7^{\circ}$ (range, $4^{\circ}$ to $11^{\circ} ; \mathrm{SD}=2 ; \mathrm{p}<0.0001$ ), which is a $65.5 \%$ decrease (Table 1).

TABLE 1 - The computed tomography scan measurements of the distal tibial rotational angle in groups $\mathrm{A}$ and $\mathrm{B}$.

\begin{tabular}{|c|c|c|c|c|c|}
\hline \multirow[t]{2}{*}{$\begin{array}{l}\text { Group A } \\
\text { Animals }\end{array}$} & \multicolumn{2}{|c|}{$\begin{array}{c}\text { external rotation } \\
\text { angle } \\
\text { (degrees) }\end{array}$} & \multirow[t]{2}{*}{$\begin{array}{l}\text { Group B } \\
\text { Animals }\end{array}$} & \multicolumn{2}{|c|}{$\begin{array}{l}\text { external rotation } \\
\text { angle (degrees) }\end{array}$} \\
\hline & $\begin{array}{l}\text { Twisted } \\
\text { tibia } \\
\text { (right) }\end{array}$ & $\begin{array}{c}\text { Control } \\
\text { tibia } \\
\text { (left) }\end{array}$ & & $\begin{array}{c}\text { Twisted } \\
\text { tibia } \\
\text { (right) }\end{array}$ & $\begin{array}{l}\text { Control } \\
\text { tibia } \\
\text { (left) }\end{array}$ \\
\hline 1 & 5 & 22 & 16 & 4 & 23 \\
\hline 2 & 8 & 20 & 17 & 7 & 18 \\
\hline 3 & 9 & 26 & 22 & 8 & 21 \\
\hline 4 & 8 & 24 & 23 & 9 & 22 \\
\hline 5 & 7 & 24 & 24 & 9 & 27 \\
\hline 6 & 10 & 26 & 25 & 11 & 25 \\
\hline 7 & 8 & 25 & 26 & 6 & 26 \\
\hline 8 & 9 & 23 & 28 & 7 & 26 \\
\hline 9 & 7 & 22 & 29 & 6 & 21 \\
\hline 10 & 9 & 25 & - & - & - \\
\hline mean & 8 & 24 & & 7 & 23 \\
\hline $\begin{array}{l}\text { Standard } \\
\text { deviation }\end{array}$ & 1 & 2 & & 2 & 3 \\
\hline $\mathrm{p}$ & $\mathrm{p}<0$ & 001 & & $\mathrm{p}<0$ & 001 \\
\hline
\end{tabular}

When comparing the twisted tibias in group A with those in group $\mathrm{B}$, the rotation angle was not significantly different $(\mathrm{p}=0.4996)$. The result was the same when comparing the control tibias $(\mathrm{p}=0.6818)$ (Table 1$)$.

In group $\mathrm{A}$, the $\mathrm{BMD}$ of the mid-diaphysis in the control tibias averaged $0.37 \mathrm{~g} / \mathrm{cm}^{2}$ (range, $0.29 \mathrm{~g} / \mathrm{cm}^{2}$ to $0.55 \mathrm{~g} /$ $\left.\mathrm{cm}^{2} ; \mathrm{SD}=0.10\right)$. The twisted tibias had a mean BMD of $0.97 \mathrm{~g} /$ $\mathrm{cm}^{2}$ (range, $0.81 \mathrm{~g} / \mathrm{cm}^{2}$ to $1.23 \mathrm{~g} / \mathrm{cm}^{2} ; \mathrm{SD}=0.10$ ), which is a $61.8 \%$ increase in the BMD on the twisted side $(\mathrm{p}<0.0001)$ (Table 2).

TABLE 2 - Bone mineral density (BMD) measurements at the central diaphysis in groups $\mathrm{A}$ and $\mathrm{B}$.

\begin{tabular}{|c|c|c|c|c|c|}
\hline \multirow{2}{*}{$\begin{array}{l}\text { Group A } \\
\text { Animals }\end{array}$} & \multicolumn{2}{|c|}{$\operatorname{BMD}\left(\mathrm{g} / \mathrm{cm}^{2}\right)$} & \multirow{2}{*}{$\begin{array}{l}\text { Group B } \\
\text { Animals }\end{array}$} & \multicolumn{2}{|c|}{$\mathrm{BMD}\left(\mathrm{g} / \mathrm{cm}^{2}\right)$} \\
\hline & $\begin{array}{c}\text { Twisted } \\
\text { tibia } \\
\text { (right) }\end{array}$ & $\begin{array}{c}\text { Control } \\
\text { tibia } \\
\text { (left) }\end{array}$ & & $\begin{array}{c}\text { Twisted } \\
\text { tibia } \\
\text { (right) }\end{array}$ & $\begin{array}{c}\text { Control } \\
\text { tibia } \\
\text { (left) }\end{array}$ \\
\hline 1 & 1.23 & 0.29 & 16 & 0.87 & 0.75 \\
\hline 2 & 1.22 & 0.55 & 17 & 1.10 & 0.74 \\
\hline 3 & 0.92 & 0.36 & 22 & 1.25 & 0.77 \\
\hline 4 & 0.90 & 0.52 & 23 & 0.78 & 0.57 \\
\hline 5 & 0.91 & 0.32 & 24 & 0.91 & 0.84 \\
\hline 6 & 0.96 & 0.42 & 25 & 0.89 & 0.88 \\
\hline 7 & 0.81 & 0.26 & 26 & 1.25 & 0.94 \\
\hline 8 & 0.93 & 0.34 & 28 & 0.87 & 0.30 \\
\hline 9 & 0.95 & 0.38 & 29 & 0.82 & 0.48 \\
\hline 10 & 0.84 & 0.29 & - & - & - \\
\hline mean & 0.97 & 0.37 & & 0.97 & 0.70 \\
\hline $\begin{array}{l}\text { Standard } \\
\text { deviation }\end{array}$ & 0.14 & 0.10 & & 0.18 & 0.21 \\
\hline $\mathrm{p}$ & \multicolumn{2}{|c|}{$\mathrm{p}<0.0001$} & & \multicolumn{2}{|c|}{$\mathrm{p}=0.0023$} \\
\hline
\end{tabular}

In group $\mathrm{B}$, the $\mathrm{BMD}$ of the control tibias averaged 0.70 $\mathrm{g}$ (range, 0.30 to $0.94 ; \mathrm{SD}=0.10$ ). The twisted tibias had a mean BMD of $0.97 \mathrm{~g} / \mathrm{cm}^{2}$ (range, 0.78 to $1.25 ; \mathrm{SD}=0.21$ ), which is an increase of $27.8 \%$ on the twisted side $(\mathrm{p}=0.0023)$ (Table 2$)$. The BMD comparison between group A and B was not significantly different when comparing the twisted tibias $(p=0.9567)$ but was significantly different among the control tibias $(\mathrm{p}=0.0004)$, with the greater BMD in group B (Table 2).

\section{Microscopic findings}

In group $\mathrm{A}$, the cross sections of the specimens of the control bone presented a cylindrical shape with four distinct layers of lamellae. The most external layer was thinner and formed by immature bone resulting from subperiosteal ossification. The next 
layer was formed by more mature bone with organized trabecular disposition and well-defined inter-trabecular spaces. Deeper, there was a distinct layer with a thinner and a more packed and organized trabecular pattern. Finally, the inner layer was narrower and formed by younger trabeculae resulting from endosteal ossification.

In groups $\mathrm{A}$ and $\mathrm{B}$, the cross sections of the twisted bones were grossly cylindrical with asymmetry of the cortical thickness, which occurred by subperiosteal bone deposition mainly on the lateral portion of the diaphysis (Figure 3).

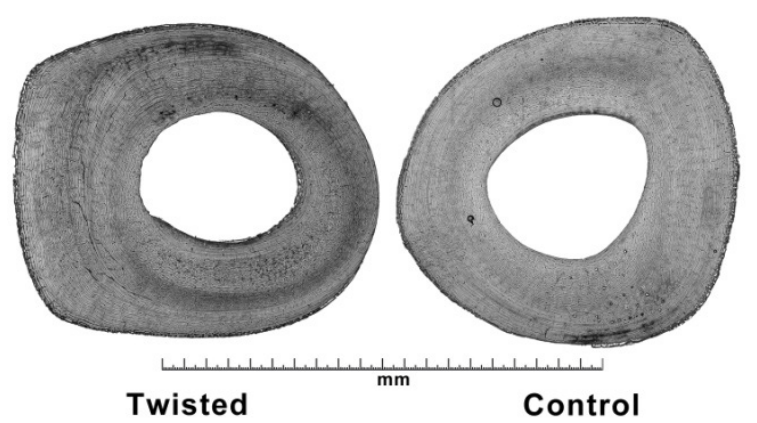

FIGURE 3 - Cross sectional micrograph at the mid-diaphysis of the twisted and control tibias in a representative animal from group A. The twisted tibia presents a different transversal shape that resulted from subperiosteal bone deposition, with thickening more pronounced on the lateral side of the bone (undecalcified specimens; toluidine blue staining).

In this newly formed bone area, the trabecular pattern in layers was more disarranged. In the twisted bone of animals belonging to group A, small cracks were found in the central layer of the transverse section, which corresponded to the original old bone (Figure 4).

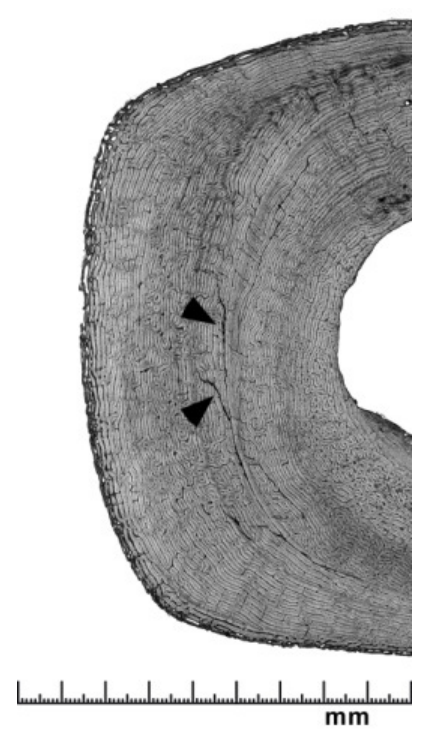

FIGURE 4 - Detail of the cortex of the twisted tibia in a representative animal from group B that illustrates the cracks located in the central bone layer (arrow heads) (undecalcified specimen; toluidine blue staining).
Polarized light examination of the cross-sections of the control tibias showed that each layer had a distinct arrangement of collagen fibers. In the twisted bone, the trabecular pattern was more disarranged and was primarily in the thicker area of the newly formed bone located on the lateral side. However, in the twisted bone group B animals, the collagen fibers were more organized than in group A but still presented some distortion (Figure 5).

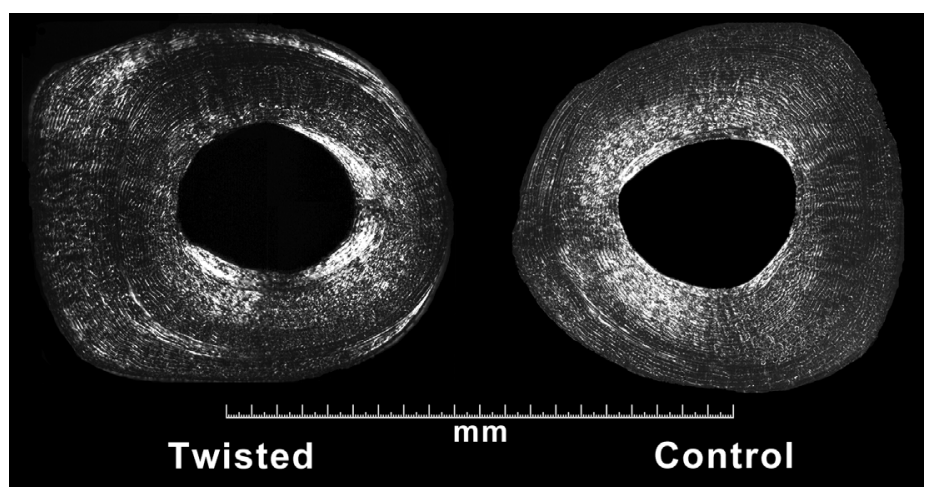

FIGURE 5 - Polarized light microscopy of transverse sections of the twisted and control tibias in a group A animal illustrating the different arrangement of the collagen fibers primarily affecting the thicker cortical region.

\section{Discussion}

Our results showed that a controlled twisting applied to the immature sheep tibia causes progressive rotation of the distal extremity, which changes the bone's rotational axis. Additionally, three months after removing the external twisting device, maintenance of the rotation of the distal tibial extremity showed that these changes were not reversed during that observation period and may persist thereafter. This result is consistent with the classical observation in orthopedics that the acquired abnormal rotational malalignment, once established, does not spontaneously correct with time even after growth.

Although the mechanical properties of the mature and immature bone have been studied extensively and a considerable number of papers on the material characteristics of aging bones have been published ${ }^{10-12}$, less information exists concerning the mechanical properties of young bones.

Immature bones are weaker and display more plastic deformation than mature bones. In terms of mechanics, in twisting, such features are manifested by a decreased maximum torque capacity and a greater angular deformation ${ }^{13,14}$.

From a clinical point of view, as a result of the increased plasticity, the immature bone displays peculiarities that reflect a pattern of failure different from that of the adult skeleton. Children's bones may present traumatic plastic deformation that cause bone 
bowing without a macroscopic fracture". The so-called "torus" fracture in children is a buckling that occurs at the metaphysis and is secondary to an acute longitudinal compression of the bone at this region. In a greenstick fracture, the bone is partially broken, but one cortex remains connected to the adjacent bone. Even when a complete fracture occurs, the fracture rarely presents with comminution of the fragments. These findings illustrate the distinctive mechanical behavior of young bone under the action of acutely applied forces. This clinical behavior is explained by the unique mechanical structure and material properties of the immature bone: the younger bone has lower modulus of elasticity, bending strength and ultimate tensile stress $^{16}$, and less mineral content ${ }^{17}$. These parameters increase with age until maturity ${ }^{18}$.

Young bone has a greater resistance to overload failure not only because of its physical size, but also because of its capability to deform more in an attempt to relieve the externally applied forces ${ }^{19}$. Being less stiff and more plastic allows the growing bone to deflect and absorb more energy before breaking ${ }^{16}$.

Based on the above-mentioned clinical and experimental findings, we assumed that a controlled progressive externally applied torsional force to an immature long bone could result in a rotational deformation that might lead to a permanent change of the bone geometry, as speculated by Moreland ${ }^{3}$. Previously, we tested the isolated immature tibias of 6-month-old lambs to establish the bone mechanical profile. As a result, we conclude that the application of $2^{\circ}$ per week of angular deformation was safe enough to assure a plastic deformation without the danger of a fracture. The only accidental fracture occurred in the control tibia in an animal belonging to group B; this fracture does not suggest any direct relation with the torsional process but, rather, it represents an accident that may occur in animals in pasture.

This experiment clearly shows that slow rotational force applied to immature lamb bones can change their transverse axis, and this change is accompanied by microscopic alterations characterized by asymmetrical deposition of bone on one side of the cortex, with an ensuing modification of the rotational axis. The small cracks observed in animals belonging to group A can be explained by the mean of energy dissipation ${ }^{19}$. These cracks also represent a way of bone remodeling that occurs as healing takes place $^{20}$. The absence of such cracks in the animals belonging to group B indicates that the remodeling process has occurred, which may have contributed to the incorporation of the acquired changes to the bone structure. The polarized light microcopy method is able to depict the reorganization of collagen fibers that, in our case, reflected the accommodation of the collagen to the new microscopic anatomy that occurred in response to the abnormal mechanical demand that the twisting process created in the bone.

Our microscopic data showed an asymmetrical deposition of bone on the medial face of the cortex and likely reflects a stress concentration on that area that stimulated bone formation by periosteal activity. In fact, bone is a mechanosensitive tissue that adapts its structure to the mechanical environment ${ }^{19}$.

The persistence of the acquired rotational alterations three months after removing the rotational device (group B) may suggest that such changes can be permanent, but long-term followup studies are necessary to confirm this assumption.

One speculative advantage of the method investigated here is that in the case of human applications, a patient could walk during the treatment, thereby facilitating the dynamic evaluation of the correction by clinical parameters, such as the foot angle progression. However, our data were obtained from the bones of healthy animals. In the case of osteopenic bones, such as those seen in young patients with cerebral palsy, the mechanical properties of the long bones are altered; therefore, they may be weaker, more brittle and less plastic than normal bone, which favors fractures. As a result, our data cannot be directly transferred to humans with altered bones, and more research focusing on this topic is required.

This study may open new possibilities to treat tibial rotational deformities with a method that appears to be safe, as it is gradual and avoids the complications that are associated with other methods acutely performed with osteotomies.

\section{Conclusion}

The response to torsion in a long bone has been clearly demonstrated as having a predictable and safe outcome.

\section{References}

1. Kristiansen LP, Gunderson RB, Steen H, Reikeras O. The normal development of tibial torsion. Skeletal Radiol. 2001;30(9):519-22.

2. Dreher T, Wolf S, Braatz F, Patikas D, Doderlein L. Internal rotation gait in spastic diplegia-critical considerations for the femoral derotation osteotomy. Gait Posture. 2007;26(1):25-31.

3. Moreland MS. Morphological effects of torsion applied to growing bone. An in vivo study in rabbits. J Bone Joint Surg Br. 1980;62$\mathrm{B}(2): 230-7$.

4. Barlow DW, Staheli LT. Effects of lateral rotation splinting on lower extremity bone growth: an in vivo study in rabbits. J Pediatr Orthop. 1991;11(5):583-7.

5. Delgado ED, Schoenecker PL, Rich MM, Capelli AM. Treatment of severe torsional malalignment syndrome. J Pediatr Orthop. 1996;16(4):484-8.

6. de Roode CP, Hung M, Stevens PM. Supramalleolar osteotomy: a comparison of fixation methods. J Pediatr Orthop. 2013;33(6):672-7.

7. Dodgin DA, De Swart RJ, Stefko RM, Wenger DR, Ko JY. Distal tibial/fibular derotation osteotomy for correction of tibial torsion: 
review of technique and results in 63 cases. J Pediatr Orthop. 1998;18(1):95-101.

8. Slawski DP, Schoenecker PL, Rich MM. Peroneal nerve injury as a complication of pediatric tibial osteotomies: a review of 255 osteotomies. J Pediatr Orthop. 1994;14(2):166-72.

9. Nolen RS. AVMA board approves Panel on Euthanasia report: updated guidelines cover more species and methods. J Am Vet Med Assoc. 2011;239(10):1269.

10. Vogel HG. Influence of maturation and aging on mechanical and biochemical parameters of rat bone. Gerontology. 1979;25(1):16-23.

11. Evans FG. The mechanical properties of bone. Artif Limbs. 1969;13(1):37-48.

12. Evans FG. Mechanical properties and histology of cortical bone from younger and older men. Anat Rec. 1976;185(1):1-11.

13. Torzilli PA, Takebe K, Burstein AH, Heiple KG. Structural properties of immature canine bone. J Biomech Eng. 1981;103(4):232-8.

14. Torzilli PA, Takebe K, Burstein AH, Zika JM, Heiple KG. The material properties of immature bone. J Biomech Eng. 1982;104(1):12-20.

15. Borden S. Traumatic bowing of forearm in children. J Bone Joint Surg Am. 1974;56(3):611-6.

16. Hirsch C, Evans FG. Studies on some physical properties of infant compact bone. Acta Orthop Scand. 1965;35(4):300-13.

17. Currey JD, Butler G. Mechanical-properties of bone tissue in children. J Bone Joint Surg Am. 1975;57(6):810-4.

18. Keller TS, Spengler DM. Regulation of bone stress and strain in the immature and mature rat femur. J Biomech. 1989;22(11-12):1115-27.

19. Turner CH. Bone strength: current concepts. Ann N Y Acad Sci. 2006;1068:429-46.

20. Burr DB. Why bones bend but don't break. J Musculoskelet Neuronal Interact. 2011;11(4):270-85.

\section{Correspondence:}

Jose Batista Volpon

Faculdade de Medicina de Ribeirão Preto

Avenida Bandeirantes, 3900

14048-900 Ribeirão Preto - SP Brasil

hc.ortopedia@gmail.com

Received: Nov 18, 2013

Review: Jan 20, 2014

Accepted: Feb 21, 2014

Conflict of interest: none

Financial source: National Council of Technological and Scientific Development (CNPq) (480390/2012-6 and 310206/2011-1)

${ }^{1}$ Research performed at Bioengineering Laboratory, Department of Biomechanics, Medicine and Rehabilitation of the Locomotor System, School of Medicine of Ribeirao Preto, University of Sao Paulo (USP), Brazil. 